\title{
Four-Wave Mixing in Optical Phase Conjugation System with Pre-Dispersion
}

\author{
Abdallah A. I. Ali*, Christian S. Costa, Mohammad A. Z. Al-Khateeb, Filipe M. Ferreira, and Andrew D. Ellis \\ Aston Institute of Photonic Technology, Aston University, Aston Triangle, Birmingham, B4 7ET, UK \\ Email: *aliaai@aston.ac.uk
}

\begin{abstract}
We mathematically analyze Kerr-induced fiber nonlinearity in an optical communication system using mid-link optical phase conjugation and pre-dispersion. The derived analytical expressions are used to describe the system performance and show great matching with simulations. Furthermore, based on these expressions, we predict the performance of a transmission system and prove a 3dB improvement in Q-factor compared to the OPC system without pre-dispersion.
\end{abstract}

\section{INTRODUCTION}

Optical Phase conjugation (OPC) is an all optical method for simultaneous mitigation of chromatic dispersion and nonlinearity impacts in single mode fiber (SMF). It is based on reversing the phase of the transmitted signal at the middle of the link, while dispersion and Kerr-induced nonlinear effects are compensated for during propagation in the second half of the link [1]. However, in order to achieve perfect dispersion and nonlinearity compensation using mid-link OPC, the power profile and dispersion need to be symmetric with respect to the OPC. One method to achieve the power symmetry is using short span lengths which means closely spaced amplifiers and further cost, and energy consumption [2]. Another approach where the Raman amplification can be also used to enhance the power symmetry [3].

In this context and as an alternative, Minzioni et. al. proposed in [4] the addition of suitable dispersive elements before or after OPC to enhance the power symmetry only in the regions of the link where signals propagate with higher power (right after the lumped amplifiers). From graphical analysis, they concluded that the maximum compensating efficiency occurs for using dispersive elements with accumulated dispersion of $-D\left(L-L_{e f f}\right)$, where $\mathrm{D}$ and $\mathrm{L}$ are the transmission fiber dispersion coefficient and span length respectively and $L_{e f f}$ is the effective length. In this paper, to the best of our knowledge, for the first time, signal-signal nonlinearity in mid-link OPC system with lumped amplification and pre-dispersion before OPC has been mathematically analyzed. The analytical result has been validated through simulation. In addition, we have used the analytical formula to predict the performance of a transmission system and the results show that the Q-factor of the transmission of 224Gbps PM-16QAM system over 10 spans of $100 \mathrm{~km}$ SMF has been improved by $3 \mathrm{~dB}$ due to the pre-dispersion and fully in accordance with the analytical predictions.

\section{Four-Wave MiXing in Pre-Dispersed OPC System}

To analyze the four-wave mixing (FWM) power generated in mid-link OPC system with lumped amplification and predispersion, we assume, a system with $N$ spans, length $L$, dispersion $\beta_{2}$ and attenuation $(\alpha)$, which is completely compensated with an amplifier after each fiber span. An ideal phase conjugator is placed at the middle of the link and an extra dispersion compensation fiber (DCF) is added before the OPC. The nonlinearity from DCF is ignored for simplicity. Using the conventional approach [5] after considering the effect of multiple spans, OPC and DCF, an analytical formula for the idler power from three signal frequencies $f_{1}, f_{2}$, and $f_{3}$ with powers $P_{1}, P_{2}$, and $P_{3}$ respectively can be written as,

$$
\begin{gathered}
P_{f}=\frac{4 D_{g} \gamma^{2} P_{1} P_{2} P_{3}}{9\left(\alpha^{2}+\Delta \beta^{2}\right)^{2}} \frac{\sin ^{2}(N \Delta \beta L / 4)}{\sin ^{2}(\Delta \beta L / 2)} \kappa^{2} \\
\kappa=\alpha\left(e^{-\alpha L} \sin \left[\left(\Delta \beta L-\Delta \beta_{1} L_{1}\right) / 2\right]+\sin \left[\left(\Delta \beta L+\Delta \beta_{1} L_{1}\right) / 2\right]\right) \\
+\Delta \beta\left(e^{-\alpha L} \cos \left[\left(\Delta \beta L-\Delta \beta_{1} L_{1}\right) / 2\right]-\cos \left[\left(\Delta \beta L+\Delta \beta_{1} L_{1}\right) / 2\right]\right) .
\end{gathered}
$$

Where $\gamma$ and $\alpha$ are the nonlinear coefficient and attenuation factor of SMF respectively. $L_{1}$ is the length of DCF and $\Delta \beta$ is the phase mismatch term between the three signals in the transmission fiber and for standard SMF can be approximated by considering only the second order dispersion $\beta_{2}$ as, $\Delta \beta=-$ $4 \pi^{2} \beta_{2}\left(f_{1}-f_{3}\right)\left(f_{2}-f_{3}\right)$ and $\Delta \beta_{1}$ is the equivalent phase mismatch for the DCF. $D_{g}$ represents the degeneracy factor and equal to 3 if $f_{1}=f_{2}$ and 6 if $f_{1} \neq f_{2}$. Setting $L_{1}=0$ gives the FWM power for a conventional OPC system [6], and setting $k^{2}=\frac{1}{4}\left(1-e^{-\alpha L}\right)^{2}+e^{-\alpha L} \sin ^{2}\left(\frac{\Delta \beta L}{2}\right)$ and $N=(2 N)$ provides the FWM power for a system without OPC [7]. The impact of pre-dispersion is expressed through the symmetry efficiency $\kappa$, and is minimized for each combination of signal frequencies for a certain value of $\Delta \beta_{1} L_{1}$.

In order to calculate the total nonlinearity affecting the signal, we assumed that $f_{1}$ is the signal and $f_{2}$ and $f_{3}$ are interferes. The total FWM power can be calculated by double integrating of Eq. (1) over the signal bandwidth [8]. The integration was solved numerically and the signal to noise ratio (Q-factor) were calculated by dividing the signal power over the sum of amplifier linear noise and total FWM power, assuming that the nonlinear power distribution is Gaussian [8]. 


\section{Simulation Results}

\section{A. Four-Wave Mixing Power}

In order to validate Eq. (1), a simulation was run using VPITransmissionMaker 9.5 by transmitting two continuous wave $(\mathrm{CW})$ laser signals with $0 \mathrm{dBm}$ powers in $10 \times 100 \mathrm{~km}$ SMF with dispersion coefficient, attenuation and nonlinear coefficient equal to $16 \mathrm{ps} / \mathrm{nm} / \mathrm{km}, 0.2 \mathrm{~dB} / \mathrm{km}$ and 1.3 (w.km) ${ }^{-1}$ respectively. The simulation was run with 8 samples per symbol. An ideal OPC (no insertion loss or frequency conversion) was implemented in Matlab. The FWM powers were measured at the end of the link at different frequency separations $(\Delta f)$ between the two CW lasers. Figure (1) shows two different cases; no pre-dispersion (red) and pre-dispersion of oppositesign to and magnitude of $\left(L-L_{e f f}\right) D$.

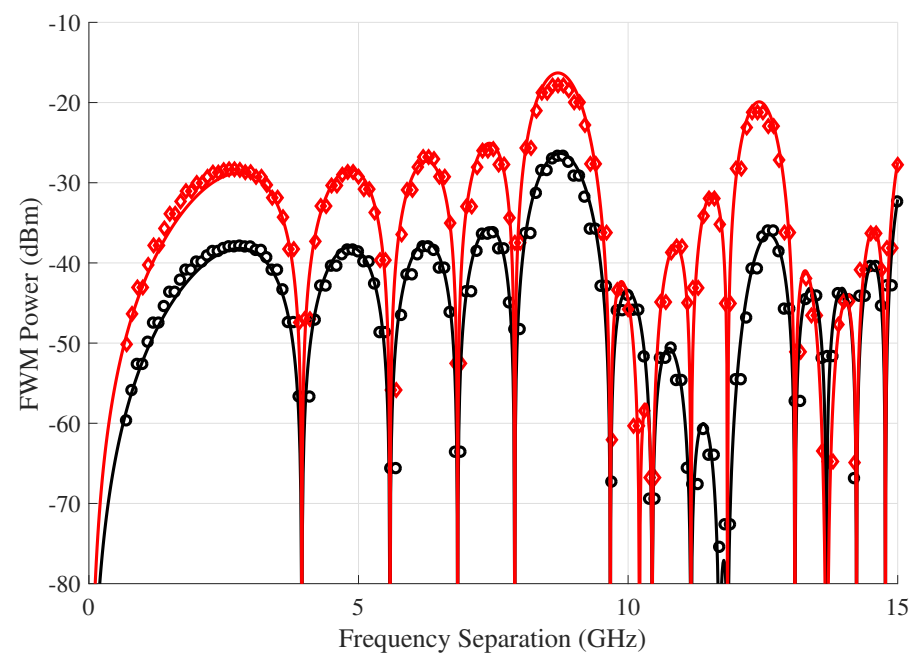

Fig. 1: FWM power of OPC system as a function of frequency separations between the two signals in two cases: without pre-dispersion (red) and with pre-dispersion (black) for theory (lines) and simulation (markers).

The solid lines represent Eq. (1) and the symbols represent the simulation results are clearly showing an excellent match between theory and simulation. Figure (1) shows that the FWM power is eliminated by the OPC in both cases in the the strongly phase matching region. For the weakly phase matched contributions, FWM power is typically suppressed by around $10 \mathrm{~dB}$ due to the in-line dispersion $1256 \mathrm{ps} / \mathrm{nm}$. In addition, the pre-dispersion affects the FWM efficiency and does not affect the phase array which cause the lopes.

\section{B. The Performance of PM-16QAM in Pre-Dispersed OPC System}

Using the same transmission link, we simulated the performance of 224 Gbps PM-16QAM operating at $193.1 \mathrm{THz}$. We used VPITransmissionMaker 9.5 for fibre transmission and Matlab for signal recovery and performance measurement. The transmitted bits was $2^{20}$ bits per polarization, and the modulated optical signals was simulated with 8 samples per symbol. The PMD and dispersion slope were ignored to focus on the nonlinearity compensation. Erbium-doped fiber amplifiers (EDFA) were used with $6 \mathrm{~dB}$ noise figure. The $\mathrm{Q}$ factor was measured from error vector magnitude (EVM). Figure (2) shows the Q-factor as a function of the fibre input power, in the two scenarios and compared theory (solid lines) with simulations (symbols).

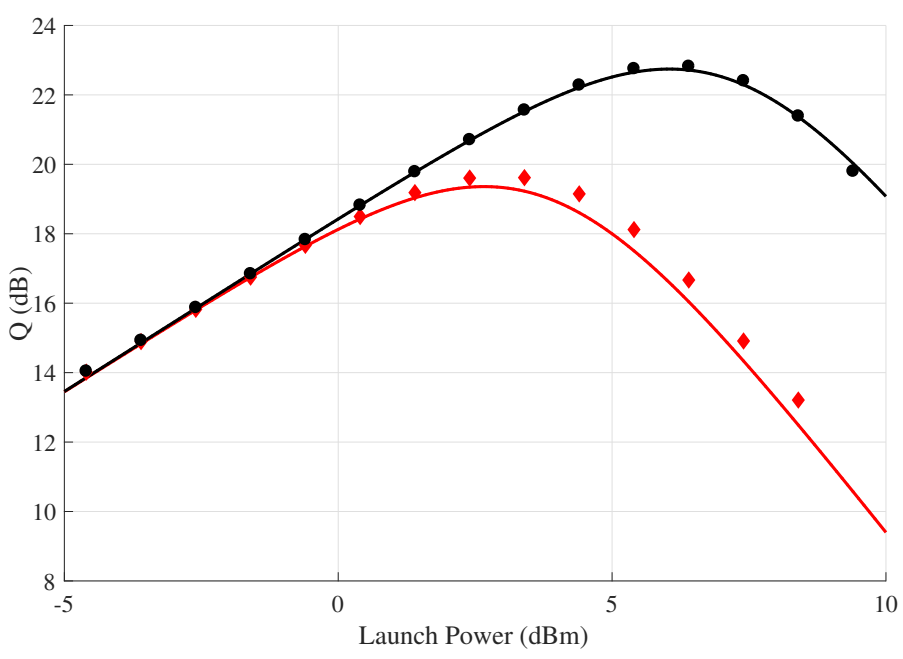

Fig. 2: The Q-factor of 224Gbps PM-16QAM transmitted over 10x100km SMF link incorporating an OPC as a function of the launched power in two cases: without pre-dispersion (red) and with pre-dispersion (black) for theory (lines) and simulation (markers).

The results show that the pre-dispersion will produce an improvement in Q-factor measured at the optimum power about $3 \mathrm{~dB}$ compared with OPC system without DCF. The simulation results closely match the theoretical curves with an error less than $0.3 \mathrm{~dB}$ measured at the optimum $\mathrm{Q}$ value. Figure (3) shows the maximum $\mathrm{Q}$ factor for different link lengths with $100 \mathrm{~km}$ span lengths and different number of spans with consistent $3 \mathrm{~dB}$ improvement due to the predispersion.

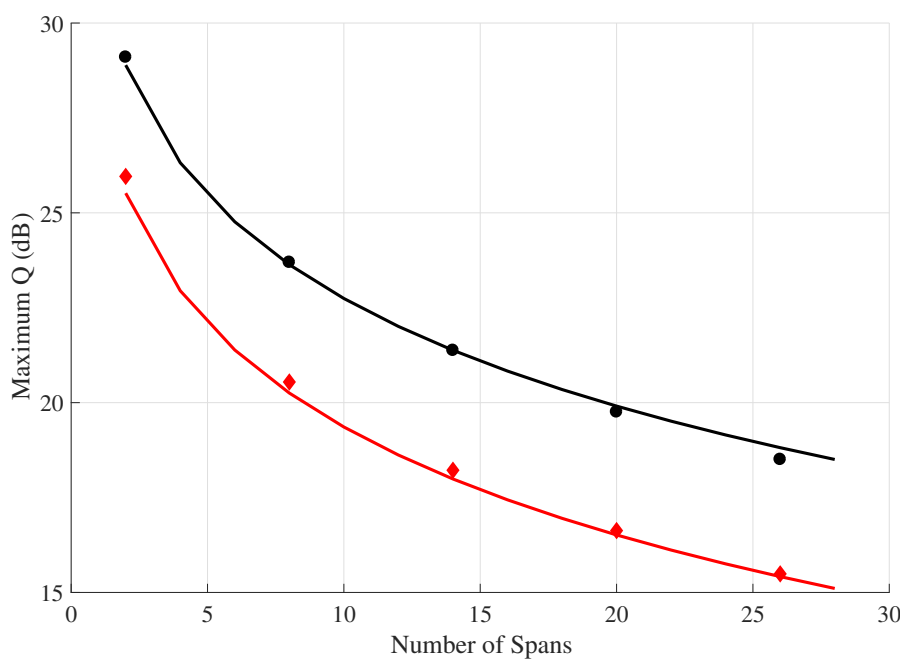

Fig. 3: The maximum $Q$ factor as a function of the link length with $100 \mathrm{~km}$ span length in two cases: without pre-dispersion (red) and with pre-dispersion (black) for theory (lines) and simulation (markers). 


\section{CONCLUSION}

An analytical expression for the FWM power in mid-link OPC system with pre-dispersion has been introduced. The analytical model has been verified through numerical simulation. In addition, it has been used to predict the performance of 224 Gbps PM-16QAM transmission system, where the results show that a Q-factor improvement of $3 \mathrm{~dB}$ can be achieved using a DCF before the OPC.

\section{ACKNOWLEDGEMENT}

This work has been partially supported by the EPSRC (Grants EP/L000091/1-PEACE, EP/J017582/1-UNLOC) and the European Union (Grants 659950-INVENTION, 654809HSPACE).

\section{REFERENCES}

[1] A. Yariv, D. Fekete, and D. M. Pepper, "Compensation for channel dispersion by nonlinear optical phase conjugation," Optics Letters, vol. 4, no. 2, pp. 52-54, 1979.

[2] N. J. Doran and A. D. Ellis, "Optical link design for minimum power consumption and maximum capacity," in Optical Communication (ECOC 2013), 39th European Conference and Exhibition on. IET, 2013, pp. 1-3.

[3] A. D. Ellis et. al., "4 Tbit/s transmission reach enhancement using 10x400 gbit/s super-channels," Journal of Lightwave Technology, vol. 34, no. 8, pp. 1717-1723, 2016.

[4] P. Minzioni and A. Schiffini, "Unifying theory of compensation techniques for intrachannel nonlinear effects," Optics express, vol. 13, no. 21, pp. 8460-8468, 2005.

[5] K. Hill, D. Johnson, B. Kawasaki, and R. MacDonald, "CW three-wave mixing in single-mode optical fibers," Journal of Applied Physics, vol. 49, no. 10, pp. 5098-5106, 1978.

[6] V. Pechenkin and I. J. Fair, "Analysis of four-wave mixing suppression in fiber-optic ofdm transmission systems with an optical phase conjugation module," Journal of Optical Communications and Networking, vol. 2, no. 9, pp. 701-710, 2010.

[7] D. Cleland, A. D. Ellis, and C. Sturrock, "Precise modelling of four wave mixing products over $400 \mathrm{~km}$ of step-index fibre," Electronics Letters, vol. 28, no. 12, pp. 1171-1173, 1992.

[8] X. Chen and W. Shieh, "Closed-form expressions for nonlinear transmission performance of densely spaced coherent optical ofdm systems," Optics Express, vol. 18, no. 18, pp. 19039-19054, 2010. 